\title{
The Relationship Between Executive Functions and Language Competences in Middle School Children
}

\author{
Boris B. Velichkovsky ${ }^{\mathrm{a},}$, Irina N. Bondarenko ${ }^{\mathrm{b}}$, \\ Varvara I. Morosanova ${ }^{b}$ \\ a Moscow State University, Moscow, Russia \\ ${ }^{\mathrm{b}}$ Psychological Institute of the Russian Academy of Education, Moscow, Russia \\ "Corresponding author. E-mail: velitchk@mail.ru
}

Background. Mastering a first language at school is mediated by the regulatory abilities of pupils. An open question is how the executive functions implementing conscious self-regulation are related to language competences.

Objective. To study the relationship between basic executive functions (switching, inhibition, working memory updating, and error correction) and language competences.

Design. A sample of 104 Russian middle school children (aged 13-15 years) performed three cognitive tasks assessing basic executive functions and two tasks assessing language competences in the areas of punctuation, spelling, morphology, syntax, semantics, vocabulary, and style.

Results. Inhibition was mostly related to punctuation, spelling, and morphology competences and was most important in the first competences task, requiring the recognition of errors. Switching was mostly related to the competences in syntax, reflecting the importance of switching attention between alternative syntactic structures. Working memory updating was the most important executive function related to language competences, with a heavy focus on higher-level lexical, semantic, and stylistic competences. The role of updating was especially important in the second competences task, which required generation of well-formed sentences. Error correction was mostly relevant for the recognition of language errors.

Conclusion. While inhibition and switching affect aspects of constructing the surface form of a sentence, working memory is preferentially related to the construction of semantically appropriate sentences. Error monitoring and correction are generally related to the recognition of language errors. Conscious self-regulation and its cognitive mechanisms are systematically related to the development of native language competences in middle school.

\section{Keywords:}

conscious self-regulation, executive functions, native language learning, language competences, secondary school 


\section{Introduction}

The problem of how a first language is mastered at school is important both theoretically and practically. The success of learning at school (learning languages in particular) may heavily depend on pupils' self-regulatory mechanisms. The relevance of studying how conscious self-regulation (SR) is related to learning success is confirmed by a large number of researches (Baumeister \& Vohs, 2004; Cascallar, Boekaerts, \& Costigan, 2006; Morosanova, Fomina, \& Bondarenko, 2015; Zimmerman \& Schunk, 2001). Morosanova, Fomina, and Bondarenko (2015) differentiate between the regulatory-personal and cognitive levels of conscious SR. The regulatory-personal level consists of personality traits that help to achieve goals. The cognitive level is represented by processes of activity planning, modeling of situational conditions, action programming, and result evaluation. The cognitive level of SR may be implemented through executive functions (EFs) - a set of meta-cognitive functions indispensable for organizing goal-directed activities in complex dynamic contexts (Diamond, 2013; Miyake et al., 2000). The purpose of the present study is to analyze the relationship between EFs and language competences in a sample of Russian middle school children.

Executive functions. Studies have demonstrated the link between EFs and effective SR of different activities, including academic learning (Hofmann, Schmeichel, \& Baddeley, 2012; Welsh \& Peterson, 2014). Miyake et al. (2000) distinguished among three "basic" EFs: switching, inhibition, and working memory updating. The switching function is related to cognitive flexibility and provides for flexible transition between tasks. Such transitions are mediated by goal changes and attention switches. Inhibition is a complex system of functions associated with a voluntary decrease in the activation of mental representations and motor responses not relevant for solving the task at hand. Inhibition plays an important role in the organization of purposeful behavior and self-control. Working memory updating is associated with the storage and processing of information necessary to solve a task. Working memory updating may play an important role in the planning of activities and in the storage of action plans and situational mental models. In addition to these basic EFs, the error monitoring and correction EF associated with ensuring the quality of cognitive activity is also of great importance for goal achievement (Dutilh et al., 2012).

Language competences. The concept of language competences (LCs) has long been a topical issue in the field of teaching both native and foreign languages. The LC concept is associated with the understanding of a language as a system and with the acquisition of language norms (Bozhovich, 2016; Kecskes, Sanders, \& Pomerantz, 2018). The assessment of LCs is a complex problem. Average school marks and exam scores give only very general information about a student's LCs. In this study, we rely on Bozhovich's (2016) approach to LCs diagnostics, which is widely used in Russia.

Relationships between EF and LC. Researchers have often suggested a relationship between EFs and language (Marslen-Wilson \& Tyler, 2007; Veraksa, Bukhalenkova, \& Kovyazina, 2018). Studies on cognitive factors of language learning (e.g., Gooch et al., 2016) suggested that EFs are related to native and foreign language learning at school. However, a recent Russian study on the contribution of EF to na- 
tive language learning in school (Verbitskaya, Malykh, Zynchenko, \& Tikhomirova, 2015) failed to find a link between an EF (working memory) and language exam grades. In any case, specific links between EFs and different LCs (lexical, syntactic, etc.) have not been sufficiently investigated yet.

\section{Method}

Hypotheses. We hypothesized the existence of significant relationships between EFs and LCs characterizing middle school pupils' success in learning the Russian language. We also assumed that there is a specificity of regulatory predictors of individual LCs (orthography, punctuation, lexical features, etc.).

Sample. The study was performed on a sample of middle school students from Moscow and Moscow Region aged 13-16 years: seventh graders $(\mathrm{N}=53$, mean age $13 \pm 0.5$ years $)$ and ninth graders $(\mathrm{N}=51$, mean age $15 \pm 0.5$ years $)$. Gender was distributed almost evenly within the sample (50.3\% female).

EF measures. We used three standard tasks for the assessment of basic EFs (Miyake et al., 2000). To assess inhibition we used the Eriksen Flanker task. The stimuli are five horizontally arranged black arrows presented against a white background in two conditions: a congruent condition $(>>>>>,<<<<<)$ and an incongruent condition $(>><>>,<<><<)$. The subjects' task is to attend to the arrow in the middle and to indicate its direction by pressing the corresponding key (" $\mathrm{z}$ " for left and "/" for right). A training session comprised of 36 trials is included. The main series contains four blocks with 36 unique trials in each. The maximum response time is $1,500 \mathrm{~ms}$. The response to stimulus interval is fixed at $1,000 \mathrm{~ms}$. Four response parameters are registered: average reaction time, percentage of correct answers, and the difference in reaction time and accuracy between the congruent and noncongruent trials (the interference effect).

To assess switching, we used the Letter-Number task with predictable task changes. A white screen is shown divided into four quadrants. In each quadrant, a pair of symbols is presented clockwise, starting with the upper left quadrant: a digit and a letter. The subjects' task is to determine whether the digit is even or odd if the characters are located in one of the upper quadrants, and whether the letter is a consonant or a vowel if the symbols appear in one of the lower quadrants. The answer is given by pressing a key (" $\mathrm{z}$ " for odd digits and vowel letters and "/" for even digits and consonant letters). The training series consists of 24 letter-digit pairs. The main series consists of 128 trials. The stimuli remain on the screen until the response is given. The response to stimulus interval is $500 \mathrm{~ms}$. Six response parameters are registered: average reaction time and accuracy, repetition trials' reaction times and accuracy, switching trials' reaction times and accuracy, and two switch costs indicating switching efficiency (the differences in reaction time and accuracy between switching and repetition trials).

To evaluate working memory updating, we used the N-Back task. The digits from 1 to 8 are presented in a pseudo-random order. The task of the subject is to answer quickly and correctly whether the currently presented digit coincides with the digit presented two positions before (2-back). The training series contains 32 trials, and the two main series each contain 48 trials. Each figure appears six times in each series (four times in the training series), once as a target. Stimulus presen- 
tation time is $500 \mathrm{~ms}$. The inter-stimulus interval is $2,000 \mathrm{~ms}$. The answer is given by pressing a key ("/" for yes or " $\mathrm{z}$ " for no). Average reaction time, accuracy, and reaction times and counts for different response types (hits, correct rejections, false alarms, and misses) are recorded.

To assess error correction, we computed the post-error slowing (PES) effect. PES (Dutilh et al., 2012) is the effect of trials after an incorrect trial exhibiting larger reaction times. The PES effect is associated with the activity of the conscious error monitoring and correction system within the anterior cingulate cortex (Botvinick, Braver, Barch, Carter, \& Cohen, 2001). We computed PES by subtracting the average reaction time from the average reaction time in the post-error trials in each EF task.

LC measures. We used two tasks for LC diagnostics (Bozhovich, 2016). Task 1 consists of 20 sentences presented in printed form. It includes eight types of errors: orthographical (spelling errors), punctuation (a sign at the end of a sentence), morphological (incorrect word forms), lexical (violation of the norms of word usage), syntactical (violation of the connection between words), semantic-syntactical (use of structure that does not correspond to the content of sentence), semantic (the content of the sentence does not correspond to the non-linguistic reality), and stylistic errors (inadequate choice of words, sentence structure, word order, word combinations, etc.). Each sentence contains one error. Each type of error is contained in two sentences. There is no more than one error in a sentence, so there are 16 sentences with errors and four sentences without errors, serving as distracters. The subject's task is to find errors in the sentences and to mark them on the sheet.

Task 2 requires active transformation of language elements. Task 2 also consists of 20 sentences; 16 sentences contain the eight types of errors from Task 1. Four sentences do not contain errors, similar to Task 1 . The subject's task is to copy the sentence to the answer sheet if there are no errors; if there is an error, the subject should correctly rewrite the sentence. No time limits were imposed in Tasks 1 and 2 . The collected data are compared with an answer key and error omissions are evaluated according to the "cost" of an error: orthographical and punctuation errors add three points to the LC score; morphological, syntactical, syntactic-semantic, semantic, lexical, and stylistic errors add two points; and "false alarms" (wrongly recognized errors) add one point. Errors made in the rewritten sentences are indicated as an additional measure, according to the type of error. Overall LC scores (computed separately for Task 1 and 2) range between zero and 60. Within each LC task, a separate score is computed for each competence (given by error type). A higher LC score is indicative of less competent language use.

Procedure. The pupils performed tasks for the assessment of LC in the classroom. Computerized tasks for EF assessment were performed in the computer lab on another day.

\section{Results}

To test the hypothesis about the relationship between EFs and LCs, we conducted a correlation analysis (Pearson's $r$ ) of all studied variables for Task 1 (Table 1) and Task 2 (Table 2). In the tables, only EF measures with significant correlations with LCs are shown. 
EF-LC relationship (Task 1). For switching, the only significant correlation is found for syntax errors. The lower the switch cost (the more effectively a child switches between tasks), the more syntax errors occur. This is contrary to intuition, and we would speak about the phenomenon of "excessive cognitive control". Excessive cognitive flexibility means excessive distractibility, and excessive attention switching during the analysis of syntactically complex constructions may provoke syntax errors. This suggests a very complex relationship between EFs and LCs in children, in whom EFs are not fully developed until the age of about 18-20 years (Luna, 2009).

Table 1

Correlations Among EF and LC Measures in Task 1

Errors

\begin{tabular}{|c|c|c|c|c|c|c|c|c|}
\hline & $\mathbf{O}$ & $\mathbf{P} \quad \mathbf{M}$ & SS & Syn & St & $\mathbf{L}$ & $S$ & OS \\
\hline \multicolumn{9}{|l|}{ Switching } \\
\hline Switch cost (errors) & & & & $-.22^{\star}$ & & & & \\
\hline \multicolumn{9}{|l|}{ Inhibition } \\
\hline Accuracy (all trials) & & & & $-.20^{\star}$ & $.19^{*}$ & & & \\
\hline Accuracy (congruent trials) & .17 & & & $-.19^{\star}$ & & & & \\
\hline Accuracy (incongruent trials) & & & & $-.23^{*}$ & $.20^{*}$ & & & \\
\hline Interference strength (errors) & & & & $.30^{* * *}$ & .17 & & & \\
\hline \multicolumn{9}{|l|}{ Updating } \\
\hline Reaction time & .17 & & & & $.25^{\star \star}$ & & & \\
\hline Reaction time (hits) & $.23^{\star}$ & & & & $.22^{*}$ & & .17 & .18 \\
\hline Reaction time (false alarms) & $.25^{\star *}$ & & & & & & & \\
\hline Reaction time (correct rejections) & & & & & $.23^{\star}$ & & & \\
\hline Accuracy (correct rejections) & & & & & $.26^{* *}$ & .18 & & \\
\hline
\end{tabular}

Notes. $O=$ orthographical errors. $P=$ punctuation errors. $M=$ morphological errors. $S S=$ semanticsyntactical errors. Syn $=$ syntactical errors. $S t=$ stylistic errors. $L=$ lexical errors. $S=$ semantic errors. $O S=$ overall LC score. Italicized numbers denote a tendency. ${ }^{*} p<.05 .{ }^{* *} p<.01 .{ }^{* *} p<.001$.

The analysis of inhibition found relationships with syntax and style errors. The lower the inhibition accuracy as well as the accuracy in congruent and incongruent trials, the more syntax errors are missed by the subjects. In the same vein, we found a clear direct relationship between interference strength and syntactic and stylistic errors. Less effective interference inhibition is associated with more syntactic and stylistic errors being omitted. This suggests an important role of inhibition associatively activated, but incorrect syntactic and stylistic constructions.

The most relationships with LCs in Task 1 were found for updating. Slower reactions in the updating task were associated with higher probability of missing spelling errors. This may be related to less effective transformation of a word's sound form into graphic form in working memory. Low speed and accuracy in the updating task are associated with worse processing of stylistic errors. This may be related 
to deficits in the construction of sentence surface structure in working memory. A similar mechanism may lead to the emergence of the relationship between inefficient updating and worse processing of lexical and semantic errors (related to the storage and processing of situational mental models in working memory). In general, in the relatively simple Task 1 which may mostly be solved by the application of highly automatized perceptual and memory routines, problems related to inefficient EF emerge mainly during the processing of high-level errors. This is because for high-level syntactic, semantic, or stylistic errors, the role of constructing an inner plan of the sentence in working memory is especially important. It is thus not surprising that updating demonstrates the only significant correlation with LC overall score in Task 1.

Table 2

Correlations Among EF and LC Measures in Task 2

\begin{tabular}{|c|c|c|c|c|c|c|c|c|c|}
\hline & \multicolumn{9}{|c|}{ Errors in Task 2} \\
\hline & $\mathbf{O}$ & $\mathbf{P}$ & $\mathbf{M}$ & SS & Syn & St & $\mathbf{L}$ & $S$ & OS \\
\hline \multicolumn{10}{|l|}{ Switching } \\
\hline RT & & & & & & & & $.29^{* * *}$ & \\
\hline RT (repeat trials) & & & & & & & & $.25^{\star \star}$ & \\
\hline RT (switch trials) & & $.19^{*}$ & & & & & & $.29^{* * *}$ & \\
\hline Switch cost (RT) & & $.22^{*}$ & & & & & & $.25^{\star *}$ & \\
\hline Post-error slowing & & & & $.24^{*}$ & & & $.22^{*}$ & & .18 \\
\hline \multicolumn{10}{|l|}{ Inhibition } \\
\hline Accuracy (congruent trials) & & & & & $-.20^{*}$ & & & & \\
\hline Accuracy (incongruent trials) & & & $-.21^{*}$ & & & & & & \\
\hline Interference strength (errors) & & & $.24^{* *}$ & & & & & & \\
\hline \multicolumn{10}{|l|}{ Updating } \\
\hline RT & & & & $.24^{*}$ & & & & & \\
\hline Accuracy & & -.18 & $-.21^{*}$ & & & & & -.18 & \\
\hline RT (hits) & & & & $.25^{* *}$ & & & & & \\
\hline RT (correct rejections) & & & & $.22^{*}$ & & & & & \\
\hline Number (false alarms) & & & & & & & .18 & & \\
\hline Number (correct rejections) & & & & & & $.22^{*}$ & & & \\
\hline Number (misses) & & & & & & & & .18 & \\
\hline
\end{tabular}

Notes. $O=$ orthographical errors. $P=$ punctuation errors. $M=$ morphological errors. $S S=$ semanticsyntactical errors. Syn $=$ syntactical errors. St $=$ stylistic errors. $L=$ lexical errors. $S=$ semantic errors. $O S=$ overall LC score. Italicized numbers denote a tendency. ${ }^{*} p<.05 .{ }^{* *} p<.01 .{ }^{* * *} p<.001$.

For error correction, no significant relationships with LCs were found in Task 1 . EF-LC relationship (Task 2). In Task 2, switching exhibited highly significant connections with LC in the areas of punctuation and semantics. Low switching efficiency (in particular, higher switching cost) led to an increase in the probability 
of skipping punctuation errors. This may be due to the fact that the correct use of punctuation requires parsing a sentence into syntactic-semantic units and effectively switching attention between them. Even more convincing are the links of switching efficiency with the construction of meaningful statements (semantic errors); such links were found for all indicators of reaction time in the switching task, as well as for switching costs.

For inhibition, a clear relationship with LCs was found in the areas of morphology and syntax. This relationship may occur because the choice of the correct word form for use in the generated sentence is determined by the need to suppress alternative forms of the same word. Such an analysis suggests that generation of the sentence structure includes a two-step process of activating lexical units with all their word forms and choosing a morphologically adequate one (extending the classical two-stage model of lexical access, Garret, 1980). A relationship between inhibition effectiveness and syntax errors has also been found. Actually, inhibition is the only EF related to syntax LC (replicating the results from Task 1). This result highlights the role of inhibition as a mechanism for choosing the correct syntactic construct among many alternatives.

For updating, relationships were found with several LCs in Task 2 (replicating the results from Task 1). Updating accuracy was related to LCs in the areas of punctuation and morphology. For punctuation, this may be due to the need for mental decomposition of the statement into syntactic-semantic and prosodic units in working memory. For morphology competence, this may be due to the need for mental analysis of the word components. As in Task 1, relationships were found between updating effectiveness and avoiding stylistic, lexical, and semantic errors. Working memory dynamics are also closely related to avoidance of semantic-syntactic errors. These results indicate that working memory updating is systematically associated with the basic competences in understanding and generating written speech. This is especially true for the competences in constructing meaningful sentences, possibly due to the involvement of working memory in the construction of semantic representations.

For error correction, some relationships with LC were obtained in Task 2, unlike Task 1. Increased post-error slowing in the switching task was related to worse processing of semantic-syntactic and lexical errors. This may be due to the ineffective use of the metacognitive strategy of critical evaluation of the generated solutions, when dealing with constructing the semantic structure of a sentence.

Data reduction. To check the hypothesis about the specifics of the regulatory predictors of different LCs, we reduced the number of analyzed variables. Exploratory factor analysis revealed latent factors characterizing the basic regulatory components that may contribute to success in mastering the Russian language in middle school. To this end, we factorized all EF indicators using the method of principal components with VARIMAX rotation. Based on the Kaiser criterion (factor weights $>1$ ), a factor solution was obtained with eight factors explaining $76 \%$ of the variance (Table 3 ):

The first three factors reflect the speed of performance in the three EF tasks. The first factor (12\% of the variance) was named "Updating Speed" (F1). The second factor (12\% of the variance) was named "Switching Speed" (F2). The third factor (11\% of the variance) was named "Inhibition Speed" (F3). 
Table 3

Factor Analysis for EF Measures

\begin{tabular}{|c|c|c|c|c|c|c|c|c|}
\hline \multirow{2}{*}{ Indicators } & \multicolumn{8}{|c|}{ Factors } \\
\hline & 1 & 2 & 3 & 4 & 5 & 6 & 7 & 8 \\
\hline $\mathrm{U} / \mathrm{RT}$ & .942 & & & & & & & \\
\hline U/RT (CRs) & .916 & & & & & & & \\
\hline U/RT (hits) & .829 & & & & & & & \\
\hline U/RT (misses) & .697 & & & & & & & \\
\hline U/RT (FAs) & .521 & & & & & & & \\
\hline S/RT (switch) & & -.989 & & & & & & \\
\hline S/RT & & -.981 & & & & & & \\
\hline S/Cost (RT) & & -.866 & & & & & & \\
\hline S/RT(repeat) & & -.797 & & & & & & \\
\hline I/RT (incongruent) & & & -.985 & & & & & \\
\hline $\mathrm{I} / \mathrm{RT}$ & & & -.973 & & & & & \\
\hline I/RT(congruent) & & & -.870 & & & & & \\
\hline I/Interference (RT) & & & -.491 & & & & & \\
\hline I/ACC (incongruent) & & & & .954 & & & & \\
\hline I/ACC & & & & .951 & & & & \\
\hline I/ACC (congruent) & & & & .408 & & & & \\
\hline I/Interference (errors) & & & & -.888 & & & & \\
\hline S/ACC & & & & & .967 & & & \\
\hline S/ACC (repeat) & & & & & .936 & & & \\
\hline S/ACC (switch) & & & & & .928 & & & \\
\hline $\mathrm{S} / \mathrm{PES}$ & & & & & .334 & & & \\
\hline U/ACC & & & & & & .935 & & \\
\hline U/N (Hits) & & & & & & .660 & & \\
\hline U/N (Misses) & & & & & & -.830 & & \\
\hline $\mathrm{U} / \mathrm{N}(\mathrm{CR})$ & & & & & & & .776 & \\
\hline $\mathrm{U} / \mathrm{PES}$ & & & & & & & .685 & \\
\hline I/PES & & & & & & & & .570 \\
\hline S/Cost (Errors) & & & & & & & & .753 \\
\hline
\end{tabular}

Notes. $U=$ Updating. $S=$ Switching. $I=$ Inhibition. $R T=$ Reaction time. ACC $=$ Accuracy. $P E S=$ Post-error slowing. $C R=$ Correct rejections. Cost (Errors) $=$ Error-related switch cost. 
The following three factors reflect the accuracy of performance of the EF tasks. The fourth factor (11\% of the variance) characterizes the accuracy of inhibition ("Inhibition Accuracy", F4). The fifth factor (10\% of the variance) consisted of switching accuracy and switching cost ("Switching Accuracy", F5). The sixth factor (9\% of the variance) included indicators of the updating task accuracy ("Updating Accuracy", F6).

The last two factors were mostly related to aspects of error correction. The seventh factor (5\% of the variance) included PES and the number of correct rejections in the updating task ("Error Correction 1", F7). The eighth factor (5\% of the variance) included PES in the inhibition task and the accuracy-related switching cost ("Error Correction 2", F8).

Similar analyses were performed separately for LC measures for each of the two LC tasks. For Task 1, a three-factor solution was obtained (63\% of the variance explained, Table 4$)$. The first factor (37\% of the variance) reflects the general command of Russian, richness of vocabulary, and the ability to apply it depending on the situation. This factor was called "Language Proficiency". The second factor (15\% of the variance) includes competences in morphology, spelling, and syntax, that is, all the language components that refer to structural analysis at the levels of words, word phrases, and sentences. This factor was called "Structure Analysis". The third factor (11\% of the variance) includes competences in orthography and punctuation and is associated with applying relevant formal rules studied at school. We named it "Literacy".

Table 4

Factor Analysis for LC Measures

\begin{tabular}{lccc} 
& \multicolumn{3}{c}{ Factors } \\
& \multicolumn{1}{c}{ Competencies } & $\mathbf{2}$ & $\mathbf{3}$ \\
\hline Stylistic & .835 & & \\
Lexical & .833 & & \\
Overall score & .715 & & \\
Syntax & & .837 & .757 \\
Orthography 1 & & .761 & -.554 \\
Morphological & & .758 & \\
Punctuation & & & \\
Orthography 2 & & & \\
\hline
\end{tabular}

Notes. Orthography $1=$ Errors made while performing Task 1 . Orthography $2=$ Errors made while reproducing sentences in Task 1.

For Task 2, a four-factor solution was obtained (64\% of the variance explained, Table 5). The first factor ( $27 \%$ of the variance) is similar to the first factor in Task 1 ("Language Proficiency"). The second factor (15\% of the variance) is similar to the third factor of Task 1, as it includes punctuation and spelling errors and reflects 
the literacy level ("Literacy Level"). The last two factors are a split of the second ("structural") LC factor in Task 1 and reflect the ability to structurally analyze units of language. Thus, the third factor (12\% of the variance) includes morphological and spelling errors - i.e., it is associated with analysis of the word structure ("Word Structure"). The fourth factor (10\% of the variance) addresses the ability to make up a well-formed sentence ("Sentence Structure").

Table 5

Factor Analysis for LC Measures

\begin{tabular}{lcccc}
\hline \multicolumn{1}{c}{ Competencies } & \multicolumn{3}{c}{ Factors } & \\
& $\mathbf{1}$ & $\mathbf{2}$ & $\mathbf{3}$ & $\mathbf{4}$ \\
\hline Stylistic & .820 & & & \\
Lexical & .796 & & & \\
Overall score & .750 & & & \\
Punctuation 1 & & .767 & & \\
Punctuation 2 & & .721 & & .854 \\
Orthography 1 & .633 & & .607 \\
Morphological & & & & \\
Orthography 2 & & & & \\
Semantic & & & & \\
Syntax & & & & \\
\hline
\end{tabular}

Notes. Orthography 1, Punctuation 1 = Errors made when performing Task 2.

Orthography 2, Punctuation 2 = Errors made in reproducing sentences in Task 2.

Regression analyses. To assess the effects of EFs on LCs, a series of multiple linear regressions was carried out. The LC factors were used separately as criteria, and the EF factors as predictors. The regressions were constructed separately for LC factors obtained for Tasks 1 and 2. To control for demographic effects, gender and age were included in the regression equations. We also included the annual grade in literature to control for general reading ability and linguistic experience. Regression coefficients for significant predictors for each of the LC factors are shown in Table 6.

In Task 1, the LC factors are systematically related to the EF factors. General language proficiency was predicted by inhibition accuracy and error correction efficiency. Structural errors were predicted by switching speed and, again, error correction efficiency. Literacy was predicted by inhibition accuracy. In Task 1, in which automatic recognition processes are important, effective switching and inhibition play a special role as LC predictors. However, inhibition may be excessive, as it actually increases the number of semantic and literacy errors. A similar phenomenon of "excessive cognitive control" has already been observed above for cognitive flexibility. Also, the regression analyses of the LC factors in Task 1 demonstrate a distinct influence of error control on LC, especially in the recognition of well-formed sentences. 
Table 6

Regression Analyses for EFs as Predictors of LCs

\begin{tabular}{|c|c|c|c|c|c|c|c|c|}
\hline $\begin{array}{c}\text { LC } \\
\text { Tasks }\end{array}$ & $\begin{array}{c}\text { Compe- } \\
\text { tencies }\end{array}$ & $\begin{array}{l}\text { Predic- } \\
\text { tors }\end{array}$ & $\mathbf{R}^{2}$ & $\beta$ & SE & B & $\mathbf{t}(92)$ & $\mathbf{p}$ \\
\hline \multirow{8}{*}{ Task 1} & \multirow{3}{*}{$\begin{array}{l}\text { Language } \\
\text { Proficiency }\end{array}$} & Age & \multirow{3}{*}{$.17^{*}$} & $-0,233$ & 0,102 & $-0,215$ & $-2,290$ & 0,024 \\
\hline & & F4 & & 0,164 & 0,097 & 0,164 & 1,685 & 0,095 \\
\hline & & F7 & & 0,210 & 0,095 & 0,210 & 2,214 & 0,029 \\
\hline & \multirow{3}{*}{$\begin{array}{l}\text { Structural } \\
\text { errors }\end{array}$} & Sex & \multirow{3}{*}{$.19^{*}$} & 0,224 & 0,098 & 0,341 & 2,292 & 0,024 \\
\hline & & $\mathrm{F} 2$ & & $-0,204$ & 0,095 & $-0,156$ & $-2,153$ & 0,034 \\
\hline & & F8 & & $-0,176$ & 0,097 & $-0,135$ & $-1,814$ & 0,073 \\
\hline & \multirow[t]{2}{*}{ Literacy } & Sex & \multirow{2}{*}{.10} & 0,174 & 0,102 & 0,347 & 1,707 & 0,091 \\
\hline & & $\mathrm{F} 4$ & & 0,200 & 0,100 & 0,200 & 2,003 & 0,048 \\
\hline \multirow{9}{*}{ Task 2} & \multirow{4}{*}{$\begin{array}{l}\text { Language } \\
\text { Proficiency }\end{array}$} & Class & \multirow{4}{*}{$.20^{*}$} & $-0,245$ & 0,100 & $-0,106$ & $-2,449$ & 0,016 \\
\hline & & $F 1$ & & 0,162 & 0,098 & 0,070 & 1,659 & 0,101 \\
\hline & & $\mathrm{F} 2$ & & $-0,226$ & 0,098 & $-0,097$ & $-2,317$ & 0,023 \\
\hline & & F6 & & $-0,178$ & 0,098 & $-0,077$ & $-1,821$ & 0,072 \\
\hline & Literacy & LN & $.13^{\star * *}$ & $-0,391$ & 0,096 & $-0,428$ & -4.057 & 0,000 \\
\hline & \multirow{2}{*}{$\begin{array}{l}\text { Word } \\
\text { Structure }\end{array}$} & F6 & \multirow{2}{*}{.12} & $-0,167$ & 0,100 & $-0,167$ & $-1,675$ & 0,097 \\
\hline & & F7 & & $-0,164$ & 0,099 & $-0,164$ & $-1,647$ & 0,103 \\
\hline & \multirow{2}{*}{$\begin{array}{l}\text { Sentence } \\
\text { Structure }\end{array}$} & Age & \multirow[b]{2}{*}{$.18^{*}$} & 0,273 & 0,102 & 0,391 & 2,673 & 0,009 \\
\hline & & F2 & & $-0,267$ & 0,099 & $-0,189$ & $-2,694$ & 0,008 \\
\hline
\end{tabular}

Notes. F1 = Updating Speed. F2 = Switching Speed. F4 = Inhibition Accuracy. F6 = Updating Accuracy. F7 = Error Correction 1. F8 = Error Correction 2. LN = Annual Grade in Literature. Italics denotes a tendency, ${ }^{*} p<.05 .{ }^{* *} p<.01 .{ }^{* *} p<.001$.

In Task 2, there are also a number of systematic relationships between LC and EF factors. Especially pronounced are the relationships between the EF factors and language proficiency. This is not surprising, since this factor includes high-level linguistic indicators associated with semantically correct organization of sentences. Semantically correct sentence construction is associated with switching efficiency and with working memory updating. The relationship of switching to these LCs has already been shown when analyzing Task 1 performance. A new result was the close link of working memory updating to the semantics-related general language proficiency LC. This connection seems to be well-founded conceptually, since working memory serves as the mental repository in which various cognitive representations are constructed and processed (Hofmann, Schmeichel, \& Baddely, 2012) and should thus be involved in the construction of the semantic structure of a sentence.

Relationships with EF factors were also obtained for both structural LC factors in Task 2. For word structure, links were found with updating factors (F6 and F7), replicating the results of the correlation analysis. This relationship may be due to 
the analysis of word components and the application of spelling rules when using working memory. For the sentence structure factor, a relationship was found with switching efficiency. For Task 1, we also found a relationship between switching efficiency and structural LC. The association between switching and the construction of syntactically well-formed sentences may be produced by effective switching, promoting a flexible choice between alternative syntactic structures. It is also interesting that in Task 2, contrary to Task 1, the relationship between the literacy factor and inhibition disappears. This may be because during sentence production, these LCs are highly automated and are beyond the reach of conscious control. This interpretation is confirmed by the fact that the only predictor of these LC is the annual grade in literature, indirectly reflecting the level of automation of reading and writing.

Beyond the intricate relationships between EFs and LCs found above, the actual relationships between EFs and LCs can be even more complex. Here, we attempted to show relatively basic influences of EFs on the cognitive processing of linguistic representations. On a more general level, EFs and conscious SR may be related to LCs through the organization of learning-related activities via goal setting, distractor suppression, multi-tasking management, etc. This last level is what is typically studied under the label of SR influences on academic achievement. The explication of these multi-level dependences between neurocognitive variables such as EFs, conscious SR, LCs, and academic achievement in linguistic domains will certainly require larger samples and more sophisticated research designs. However, the present results support the notion that EFs play a role in attaining linguistic competency in one's native language and, by extension, that well-developed EFs advance language learning generally. This may have implications both for structuring language courses at school and for developing necessary EF skills in school children via systematic cognitive exercise (Colzato \& Hommel, 2016).

\section{Conclusion}

The results allow us to draw a number of conclusions. First, the low-level LCs such as punctuation, spelling, and morphology, and competences in syntactic analysis, were linked with inhibition. For the recognition of errors (Task 1), inhibition was the leading EF. This indicates the important role of inhibiting alternative linguistic representations during analysis of sentences. Second, for switching, a relationship was found with punctuation and syntax errors, as well as with the general ability to generate meaningful statements. This indicates the important role of switching attention between linguistic units in the process of analyzing and constructing sentences. Third, LCs found the most numerous relationships with working memory updating. These can be traced for low-level LCs related to spelling and morphology, but are most pronounced for the higher-level LCs related to lexical, semantic, and stylistic errors. The special role of working memory in the analysis and construction of sentences may be related to the function of working memory as a system for storage and transformation of mental models, including sentences' semantic and syntactic structures. Fourth, error correction was also associated with LC, especially during automatic error recognition (Task 1). Overall, the results indicate that 
the cognitive mechanisms of conscious SR are systematically related to the mastering of one's native language in middle school.

\section{Acknowledgements}

This study was supported by RFBR under grant No. 17-29-09094, "Conscious selfregulation in the system of cognitive and non-cognitive mechanisms of success in learning Russian at school".

\section{References}

Baumeister, R.F., \& Vohs, K.D. (Eds.) (2004). Handbook of self-regulation: Research, theory, and applications. New York: Guilford.

Botvinick, M.M., Braver, T.S., Barch, D.M., Carter, C.S., \& Cohen, J.D. (2001). Conflict monitoring and cognitive control. Psychological Review, 108(3), 624-652. https://doi.org/10.1037/0033295X.108.3.624

Bozhovich, E.D. (2016). Razvitie yazykovoy kompetentcii kak psikhologicheskoi sistemy [The development of language competence as a psychological system]. Extended abstract of doctor's thesis. Moscow. (In Russian).

Cascallar, E., Boekaerts, M., \& Costigan, T. (2006). Assessment in the evaluation of self-regulation as a process. Educational Psychology Review, 18, 297-306. https://doi.org/10.1007/ s10648-006-9023-2

Colzato L.S., Hommel B. (2016). The future of cognitive training. In T. Strobach \& J. Karbach (Eds.). Cognitive training (pp. 201-211). Cham, Switzerland: Springer. https://doi. org/10.1007/978-3-319-42662-4_19

Diamond, A. (2013). Executive functions. Annual Review of Psychology, 64, 135-168. https://doi. org/10.1146/annurev-psych-113011-143750

Dutilh, G., Vandekerckhove, J., Forstmann, B.U., Keuleers, U., Brysbaert, M., \& Wagenmakers, E.-J. (2012). Testing theories of post-error slowing. Attention, Perception, and Psychophysics, 74(2), 454-465. https://doi.org/10.3758/s13414-011-0243-2

Garrett, M. F. (1980). Levels of processing in sentence production. In B. Butterworth (Ed.), Language production (Vol. 1) (pp. 177-220). New York: Academic Press.

Gooch, D., Thompson, P., Nash, H.M., Snowling, M.J., \& Hulme, C. (2016).The development of executive functions and language skills in early school years. Journal of Child Psychology and Psychiatry, 57(2), 180-187.

Hofmann, W., Schmeichel, B.J., \& Baddeley, A.D. (2012). Executive functions and self-regulation. Trends of Cognitive Science, 16(3), 174-80. http://dx.doi.org/10.1016/j.tics.2012.01.006

Kecskes, I., Sanders, R.E., \& Pomerantz, A. (2018). The basic interactional competence of language learners. Journal of Pragmatics, 124, 88-105. https://doi.org/10.1016/j.pragma.2017.10.019.

Luna, B. (2009). Developmental changes in cognitive control through adolescence. Advances in Child Development and Behavior, 37, 233-278. https://doi.org/10.1016/S00652407(09)03706-9

Marslen-Wilson, W. D., \& Tyler, L. K. (2007). Morphology, language and the brain: The decompositional substrate for language comprehension. Philosophical Transactions of the Royal Society: Biological Sciences, 362, 823-836. https://doi.org/10.1098/rsth.2007.2091

Miyake, A., Friedman, N., Emerson, M, Witzki, A., Howerter, A., \& Wager, T. (2000).The unity and diversity of executive functions and their contributions to complex "Frontal Lobe" 
tasks: A latent variable analysis. Cognitive Psychology, 41, 49-100. https://doi.org/10.1006/ cogp.1999.0734

Morosanova, V.I., Fomina,T.G., \& Bondarenko, I.N. (2015). Academic achievement: Intelligence, regulatory, and cognitive predictors. Psychology in Russia: State of the Art, 8(3), 136-157. https://doi.org/10.11621/pir.2015.0311

Veraksa, A.N., Bukhalenkova, D.A., \& Kovyazina, M.S. (2018). Language proficiency in preschool children with different levels of executive function. Psychology in Russia: State of the Art, 11(4), 115-129. https://doi.org/10.11621/pir.2018.0408

Verbitskaya, L.A., Malykh, S.B., Zinchenko, Yu.P., \& Tikhomirova, T.N. (2015). Cognitive predictors of success in learning Russian. Psychology in Russia: State of the Art, 8, 91-100. http://doi.org/10.11621/pir.2015.0408

Welsh, M., \& Peterson, E. (2014). Issues in the conceptualization and assessment of hot executive functions in childhood. Journal of the International Neuropsychological Society, 20(2), 152-156. https://doi.org/10.1017/S1355617713001379

Zimmerman, B.J., \& Schunk, D.H. (Eds.). (2001). Self-regulated learning and academic achievement: Theoretical perspectives. Hillsdale, NJ: Erlbaum.

Original manuscript received October 22, 2018

Revised manuscript accepted January 10, 2019

First published online February 15, 2019

To cite this article: Velichkovsky, B.B., Bondarenko, I.N., Morosanova, V.I. (2019). The relationship between executive functions and language competences in middle school children. Psychology in Russia: State of the Art, 12(1), 104-117. DOI: 10.11621/pir.2019.0108 